\title{
Simulation of Coherent Scattering in the STEM
}

\author{
J. S. Wall
}

Biology Dept., Brookhaven National Laboratory, Upton, NY 11973

The central tenet of mass measurement in the STEM (Scanning Transmission Electron Microscope) is the proportionality between the mass of all atoms in the path of the beam and the large angle annular detector signal. Since this is a dark field signal, from a wave optic point of view one might expect a quadratic dependence on thickness (scattered amplitude squared). However, Fertig and Rose $^{1}$ showed that the quadratic term is only important if the atoms are close together or the angular collection range of the annular detector is small. They showed how to calculate the "coherence volume" for any detector geometry. This is an ellipsoid of revolution with the long axis along the beam direction. Atoms within that volume would have enhanced scattering. Simulation software allows us to examine this question under a number of conditions closer to experiment.

We have adapted the multi-slice software of Kirkland ${ }^{2}$ to simulate STEM imaging of biological specimens. Currently we can simulate a $25 \mathrm{~nm}^{3}$ volume containing up to $10^{7}$ atoms specified by atomic number and floating point atomic coordinates. Structures can be from Biology (Protein Data Bank) or Materials Science and placed on an amorphous carbon film of any desired thickness or embedded in amorphous ice or negative stain. The probe wavefront entering the top surface of the specimen is simulated wave optically (aperture angle, wavelength, defocus and spherical aberration), propagated through the specimen using the multi-slice method, and propagated to the detector plane wave optically. There the amplitude is squared and integrated over the angles subtended by the various detectors. The entire process must be repeated for each point in the scan raster, requiring roughly $1 \mathrm{sec}$ per pixel for a moderately complicated specimen.

For comparison, the beam intensity distribution at the specimen surface can be convoluted with the scattering cross sections of all atoms in each vertical column and this incoherent intensity summed. This simple approximation normally used for STEM calculations gives a point-by-point comparison to the wave-optical simulation.

In the simulation shown we have used a FCC gold crystal clipped to give a $20 \mathrm{~nm}$ diameter sphere. This is placed on top of a $2 \mathrm{~nm}$ carbon substrate to represent specimens of the type frequently observed in the BNL STEM.

Simulations of this sort are useful to assess the feasibility of proposed experiments and interpret results. In addition, it facilitates quantitative comparison of microscope operating modes for existing and proposed instruments, supporting innovations where justifies and avoiding disappointments.

1. J.Fertig, H.Rose, (1977) Ultramicroscopy, 2, 269-279.

2. E.J.Kirkland, "Advanced Computing in Electron Microscopy", Plenum (1998).

3. Supported by USDOE and NIH P41-EB-002181. 

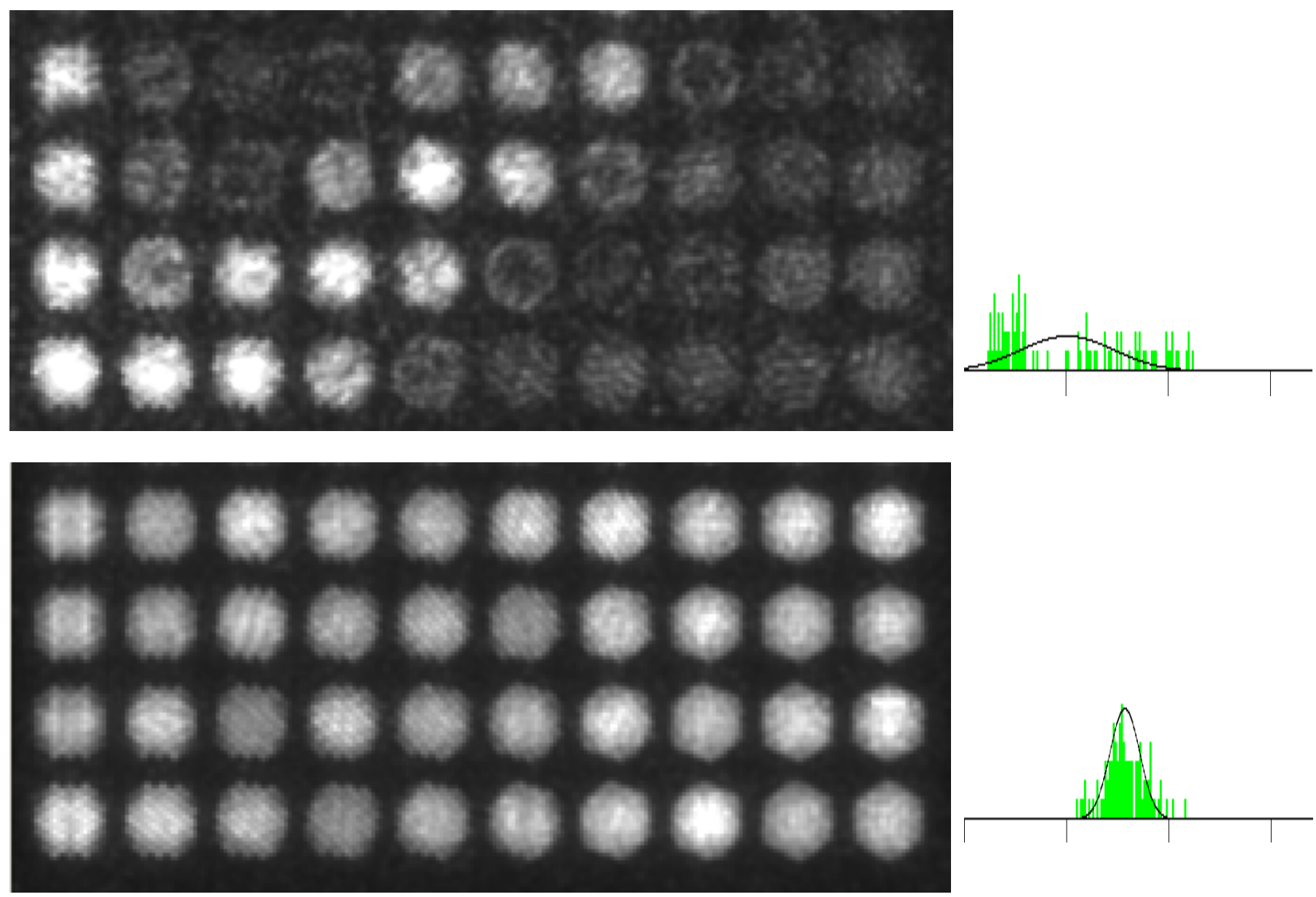

Simulated images of $2 \mathrm{~nm}$ crystalline gold spheres in various orientations. The probe energy was 40 $\mathrm{keV}$ and the aperture was $14 \mathrm{mRad}$, giving a probe size of $0.26 \mathrm{~nm}$. The upper image used the STEM small angle (SA, 15-40 mRad acceptance) annular detector and the lower used the large angle (LA, 40-250 mRad) detector. The lower left sphere in each panel corresponds to the $<100>$ orientation of the gold lattice and the balls to the right are $5 \mathrm{deg}$. rotations as if the ball were rolling on the substrate, reaching a $<110>$ orientation in the rightmost image. Rows above the bottom are initial $5 \mathrm{deg}$. rotations in the perpendicular direction prior to similar rotations. The panels to the right are histograms of the integrated intensity for all pixels within the contour of a single sphere. Note the large number of orientations where few of no diffracted beams strike the small angle detector. In such cases most of the signal comes from the edge of the particle coherent cancellation is less perfect. The large angle detector shows a much more uniform response with less dependence on orientation, as expected. In this case the distribution is well approximated by a Gaussian with $13 \%$ standard deviation. 